\title{
AN INNOVATION Application of APPROACH TO White Noise Theory RANDOM FIELDS
}


This page intentionally left blank 


\section{AN INNOVATION Application of APPROACH TO White Noise Theory RANDOM FIELDS}

\section{Takeyuki Hida}

Meijo University, Japan

$\mathrm{Si} \mathrm{Si}$

Aichi Prefectural University, Japan 
Published by

World Scientific Publishing Co. Pte. Ltd.

5 Toh Tuck Link, Singapore 596224

USA office: 27 Warren Street, Suite 401-402, Hackensack, NJ 07601

UK office: 57 Shelton Street, Covent Garden, London WC2H 9HE

\section{British Library Cataloguing-in-Publication Data}

A catalogue record for this book is available from the British Library.

\section{AN INNOVATION APPROACH TO RANDOM FIELDS - Application of White Noise Theory}

Copyright () 2004 by World Scientific Publishing Co. Pte. Ltd.

All rights reserved. This book, or parts thereof, may not be reproduced in any form or by any means, electronic or mechanical, including photocopying, recording or any information storage and retrieval system now known or to be invented, without written permission from the Publisher.

For photocopying of material in this volume, please pay a copying fee through the Copyright Clearance Center, Inc., 222 Rosewood Drive, Danvers, MA 01923, USA. In this case permission to photocopy is not required from the publisher.

ISBN 981-238-095-7

Typeset by Stallion Press

Email: enquiries@stallionpress.com

Printed in Singapore. 
To the memory of Fumi and Su Su 
This page intentionally left blank 


\section{Preface}

The purpose of this book is two-fold: First, we analyze random fields in line with innovation theory. The analysis will be carried out as an application of white noise analysis which has developed extensively in recent years. Most of the random fields to be discussed are complex systems that are parameterized by a space-time parameter $t$ or by a manifold $C$ like a curve and surface and are evolutionary with respect to those parameters. In what we discuss here, one can see, among the highlights of this volume, the effectiveness of the variational calculus applied to the random fields.

Our second aim is to show interesting applications in many areas, such as quantum dynamics, quantum field theory, statistical mechanics and molecular biology, as well as many other fields of science. There one can see significance of the innovation approach to random complex systems that are developing as the parameter varies.

The random fields that we discuss in this volume are assumed to be functionals of white noise of either Gaussian or Poisson type, parameterized by a multi-dimensional time (vector) $t$ or by a certain manifold $C$ that is running through a Euclidean space. Let a field be denoted by $X(t)=X(t, x)$ or by $X(C)=X(C, x)$, where $x$ is a sample function of a white noise and $t$ is a vector in a certain topological vector space, and where $C$ changes within a certain family $\mathbf{C}$ of smooth, closed and convex manifolds in $R^{d}$, $d \geq 1$. We focus our attention mainly on $X(C)$, where, in particular, $C$ is chosen so as to run through a space-time domain. Thus, $X(C)$ can be thought of as a realization of random complex phenomena varying as $C$ deforms and being interfered with by a fluctuation, a mathematical expression of which is a white noise $x$.

Our main interest is the determination of the probabilistic structure of $X(C)$. There are several mathematical tools for this aim; among others, we first provide its stochastic variation, denoted by $X(C)$, obtained by an 
infinitesimal deformation of the $C$. The variation therefore expresses the infinitesimal change of the random phenomenon in question. Further if we can introduce an equation for stochastic variation, then it (or its solution) characterizes the structure of $X(C)$. The study of such variational calculus is to be a sort of stochastic calculus. It might be considered an analogue of the classical theory of calculus of variation. In fact, it is partially true, but not quite. In addition to the classical results, the stochastic part of the variational calculus plays a more important role, and its study requires some advanced theory of probability.

We shall also discuss random functions depending on a function $f$. There are many good examples in these cases. It is noted that some $X(C)$ discussed above can often been reduced to this case, since $C$ may be represented by a vector-valued function.

With these assumptions, we are now ready to provide some background including the following topics which are known concepts in white noise theory:

(1) White noise with multi-dimensional parameters and with its restriction to a lower dimensional manifold. Similar facts for Poisson noise.

(2) Generalized functionals of white noise, and the $S$-transform that carries those functionals to non-random functionals of ordinary real valued functions.

(3) Theory of calculus of variations that provides a powerful tool in the analysis of random fields with the help of the $S$-transform.

(4) Infinite dimensional rotation group that describes the invariance of the white noise measure.

With this background we shall discuss stochastic variational calculus and its applications. We can see various applications that suggest to us new directions of our white noise analysis, in particular directions to quantum dynamics and quantum computation.

Before we come to the actual steps of the main subjects, we first need to determine the topology to be introduced in the class of random functions and stochastic processes, so that the variation is rigorously defined. The possible topologies are as follows:

(i) The mean square topology. In this case we start with a Hilbert space involving square integrable functionals with respect to the white noise measure. The norm of the space is given by the positive square root of the quadratic mean. The Gaussian fields have been extensively investigated within this framework. Given either Gaussian noise or other 
noise, the so called $S$-transform, mentioned in (2) above of random functional of noise is applied, so that we can use a modern version of the theory of functional analysis.

(ii) The convergence in probability. Since there are cases where the existence of moments of the random variables in question is not required, we want to discuss a sufficiently wide class of random functions, in particular random fields. Path-wise nonlinear operations are naturally introduced and discussed there. The use of characteristic functional is efficient in this case.

We are now in a position to speak of the most significant characteristics as well as the central advantages of our approach, although space is too limited to describe the details. They are listed below.

(1) If one is allowed to use intuitive notations of white noise and Poisson noise like $\dot{B}(t)$ and $\dot{P}(t)$, it is claimed that they are taken to be variables of random functions that describe the given random complex systems. As a result, we are led to introduce generalized functionals of noise. This allows us to discuss stochastic analysis for a sufficiently broad applicable area. Further, it is natural to introduce differential and integral operators in $\dot{B}(t)$ and $\dot{P}(t)$ and to establish an analysis of new kind.

(2) White noise measure enjoys rich invariance, so that the infinite dimensional rotation group can be introduced (see (4) above). This enables us to discuss a Harmonic Infinite Dimensional Analysis. Note that the analysis is essentially infinitely dimensional, the exact meaning of which will be illustrated in this book.

(3) It is emphasized that both $\dot{B}(t)$ and $\dot{P}(t)$ are elemental, being members of idealized innovation. With the factor $d t$ they can serve as random measures. On the other hand, their sample functions (generalized functions) as well as functionals of them can be dealt with within our framework. Sample function-wise stochastic properties can be investigated by this idea. For detailed calculus, we introduce a wider class of those functionals, denoted by $(\mathbf{P})$, without assuming the existence of variances. Precise characters of space-time dependency and causality of evolutional phenomena can now be discussed in a natural manner.

(4) Qualitative probabilistic properties, like stochastic optimality and reversibility, can smoothly be discussed within our framework. In particular, one can see more clearly in the case of random fields. Further, 
our setup enables us to see good connections to quantum probability and to quantum dynamics.

(5) Of course there are many applications where a person can receive a new insight and can see beautiful interplay between mathematics and other fields of science. The reader might think that this book aims mainly at some applications of mathematics, but that is not quite true. Application of mathematics only contributes little to mathematics. On the other hand, we know that applications of physics or of others to mathematics have often made vital contributions to mathematics. Part of this idea can be seen in this volume.

A final note is that most of the parts of this book are easily understood, and we may say that the book is elemental, but not elementary. However, occasionally some more difficult ideas ground this work, as the readers might guess. Thus, this book can be read on different levels, depending on the reader.

It is our regret that we did not make good connections with noncommutative geometry or some thoughts on topics related to path integrals, which can be discussed from our viewpoint. Readers will find a short note on this topic in the Epilogue at the end of this volume. Some supplementary notes related to this topic are also in the Epilogue.

T. Hida and Si Si July 2003, Nagoya, Japan 


\section{Contents}

Preface vii

1. Introduction 1

1.1 The idea of our approach . . . . . . . . . . . . . 1

1.2 Random functionals $X(C)$ depending on a manifold $C$. . 2

1.3 Stochastic variational equations . . . . . . . . . 6

1.4 Random functions $X(f) \ldots \ldots \ldots \ldots \ldots$

2. White Noise 11

2.1 Preliminaries . . . . . . . . . . . . . . . . . . 11

2.2 Multi-dimensional parameter white noise . . . . . . . . 20

2.3 Infinite dimensional rotation group $O(E) \ldots \ldots . \ldots 24$

2.4 Subgroups of $O(E) \ldots \ldots \ldots \ldots \ldots$

2.5 Laplacians . . . . . . . . . . . . . . . . . . . . 31

2.6 Invariance of white noise . . . . . . . . . . . . 31

3. Poisson Noise 33

3.1 Poisson noise functionals . . . . . . . . . . . . . . 33

3.2 Functional equations for $C_{P}(\xi) \ldots \ldots \ldots \ldots$

3.3 Observation of 1-dimensional parameter Poisson noise . . . 39

3.4 Construction of 1-dimensional Poisson noise . . . . . . . . . 41

3.5 Construction of $d$-dimensional parameter Poisson noise . . . 42

3.6 Invariance of Poisson noise . . . . . . . . . . . . 47

3.7 Multi-dimensional parameter Poisson sheet . . . . . . . 48

3.8 Compound Poisson noise . . . . . . . . . . . . . . 49

3.9 The space $(\mathbf{P}) \ldots \ldots \ldots \ldots \ldots \ldots$ 
4. Random Fields

4.1 Processes and fields as white noise functionals . . . . . . 55

4.2 Random fields $X(a)$ and $X(f) \ldots \ldots \ldots \ldots$

4.3 White noise parameterized by a point of a manifold . . . 59

4.4 Random fields parameterized by a manifold $C \ldots \ldots$. . . . 60

4.5 Random fields as white noise functionals . . . . . . . . 63

4.6 Random fields as Poisson noise functionals . . . . . . . . . . 64

5. Gaussian Random Fields $\quad 65$

5.1 A review of the canonical representations of Gaussian processes . . . . . . . . . . . . . . 65

5.2 Canonical representations of Gaussian random fields . . . . 66

5.3 Martingale. . . . . . . . . . . . . . . . 72

5.4 A review of Markov property of Gaussian processes . . . . . 73

5.5 Markov property of Gaussian random fields . . . . . . . . 77

5.6 Euclidean free field . . . . . . . . . . . . . . . . 82

6. Some Non-Gaussian Random Fields $\quad 85$

6.1 Fields of homogeneous chaos . . . . . . . . . 85

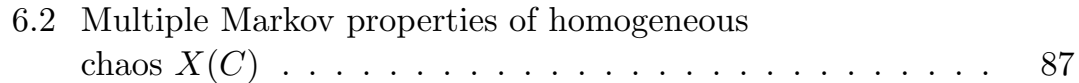

6.3 The Poisson case . . . . . . . . . . . . . . . . . . . 89

6.4 Poisson noise functionals . . . . . . . . . . . . . . 93

6.5 Random fields as Poisson noise functionals . . . . . . . . . 94

$\begin{array}{ll}\text { 7. Variational Calculus for Random Fields } & \mathbf{9 7}\end{array}$

7.1 Generalized white noise functionals and random fields . . . 97

7.2 Restriction of parameter (continued) . . . . . . . . . . 98

7.3 Variational formula for $X(C) \ldots \ldots \ldots 9$

7.4 Variational equation . . . . . . . . . . . . . 103

7.5 Existence theorem for a variational equation . . . . . . . 105

7.6 A generalization of the Ito formula for Gaussian random fields . . . . . . . . . . . . 108

7.7 The Poisson case . . . . . . . . . . . . . . . 110

7.8 Characteristic functionals . . . . . . . . . . . . . 112

8. Innovation Approach $\quad 117$

8.1 Concept of innovation . . . . . . . . . . . . . . 117

8.2 Lévy decomposition of innovation . . . . . . . . . . . . 120

8.3 Review of linear parameter case . . . . . . . . . . . . 121 
8.4 Innovations of linear processes . . . . . . . . . . . . 126

8.5 Innovation of a linear random field . . . . . . . . . . 127

8.6 Stochastic variational equations . . . . . . . . . . . . . . 129

8.7 Examples . . . . . . . . . . . . . . . . . 134

9. Reversibility 143

9.1 Reversibility of stochastic processes . . . . . . . . . . . 143

9.2 Reversibility of a random field . . . . . . . . . . . . . 146

9.3 Variational equations for quantum fields . . . . . . . . . . 149

10. Applications 151

10.1 Conformal group $C(d)$ as a subgroup of $O(E) \ldots \ldots . .151$

10.2 Spectral type of flows . . . . . . . . . . . . 153

10.3 The transversal relation . . . . . . . . . . . . . . 155

10.4 Conformal invariance of white noise . . . . . . . . 156

10.5 Action on random fields . . . . . . . . . . . 156

10.6 Mathematical biology . . . . . . . . . . . . . . . 160

10.7 Tomonaga-Schwinger equation . . . . . . . . . . . 161

$\begin{array}{ll}\text { Appendix } & 163\end{array}$

A.1 Appendix $1 \ldots \ldots \ldots \ldots \ldots$

A.2 Appendix $2 \ldots \ldots \ldots \ldots \ldots \ldots$

A.3 Appendix $3 \ldots \ldots \ldots \ldots \ldots 17 \ldots \ldots \ldots$

A.4 Appendix $4 \ldots \ldots \ldots \ldots \ldots \ldots$

$\begin{array}{ll}\text { Epilogue } & 177\end{array}$

List of Notations $\quad 179$

$\begin{array}{ll}\text { Bibliography } & 181\end{array}$

$\begin{array}{ll}\text { Index } & 187\end{array}$ 